\title{
Norois
}

Environnement, aménagement, société

249 | 2018

Patrimoine, vignoble, continuité écologique, transition énergétique, anthropologie des catastrophes

\section{Entre attachement aux lieux et gestion de la reconstruction post-sinistre : l'action municipale au centre-ville de Lac-Mégantic, Québec (Canada)}

Between place attachment and management of the post-disaster reconstruction: municipal action in downtown Lac-Mégantic, Quebec (Canada)

\section{Emmanuelle Bouchard-Bastien et Geneviève Brisson}

\section{(2) OpenEdition}

\section{Journals}

\section{Édition électronique}

URL : https://journals.openedition.org/norois/7201

DOI : 10.4000/norois.7201

ISBN : 978-2-7535-7773-2

ISSN : $1760-8546$

\section{Éditeur}

Presses universitaires de Rennes

Édition imprimée

Date de publication : 31 décembre 2018

Pagination : 75-88

ISBN : 978-2-7535-7771-8

ISSN : 0029-182X

\section{Référence électronique}

Emmanuelle Bouchard-Bastien et Geneviève Brisson, «Entre attachement aux lieux et gestion de la reconstruction post-sinistre : I'action municipale au centre-ville de Lac-Mégantic, Québec (Canada) », Norois [En ligne], 249 | 2018, mis en ligne le 31 décembre 2020, consulté le 13 janvier 2022. URL : http://journals.openedition.org/norois/7201 ; DOI : https://doi.org/10.4000/norois.7201 


\title{
Entre attachement aux lieux et gestion de la reconstruction post-sinistre : l'action municipale au centre-ville de Lac-Mégantic, Québec (Canada)
}

\author{
Between Place Attachment and Management of the Post-disaster Reconstruction: \\ Municipal Action in Downtown Lac-Mégantic, Quebec (Canada)
}

\author{
Emmanuelle Bouchard-BAstien ${ }^{a}$ et Geneviève Brisson ${ }^{b}$
}

\author{
a Institut national de santé publique du Québec - 945, avenue Wolfe, QuéBEc, Québec, Canada, G1V 5B3. \\ (emmanuelle.bouchard-bastien@inspq.qc.ca)

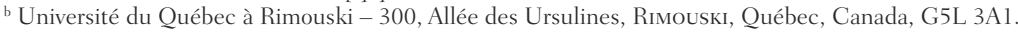 \\ (genevieve_brisson@uqar.ca)
}

\begin{abstract}
Résumé : Se basant sur une recherche qualitative réalisée à la suite de la tragédie ferroviaire de Lac-Mégantic (Québec, Canada), cet article explore la manière dont les autorités ont pris en charge la reconstruction des lieux sinistrés et prennent en compte les significations et les liens au territoire lorsqu'il est si violemment atteint par une catastrophe et par ses réaménagements. Les principaux lieux significatifs de Lac-Mégantic ont été identifiés, ainsi que les réponses mises en place par les autorités. À partir du concept de lien d'attachement au lieu et de ses modélisations, l'analyse mobilise quatre dimensions du sens aux lieux, l'identité, la fonctionnalité, l'univers social et la nature. L'étude entend offrir une contribution à l'anthropologie des catastrophes en démontrant que les lieux significatifs sont au cœur des enjeux du processus de gestion de la catastrophe et de la reconstruction à Lac-Mégantic.

Abstract: Based on qualitative research conducted after the Lac-Mégantic rail tragedy (Québec, Canada), this article explores the way in which the authorities have taken charge of the post-disaster reconstruction and take into account the meanings and links to the territory when is so violently affected by a disaster and its redevelopment. The main significant sites of Lac-Mégantic have been identified, as well as the responses put in place by the authorities. Based on the concept of place attachment and its models, the analysis mobilizes four dimensions of sense of places: place identity, place dependence, social bonding and nature bonding. The study intends to make a contribution to the anthropology of disasters by demonstrating that significant sites are at the heart of the challenges of the disaster management and reconstruction process in Lac-Mégantic.
\end{abstract}

Mots clés : gestion - centre-ville - reconstruction - sens des lieux - catastrophe - Québec (Canada)

Keywords : management - downtown area - reconstruction - sense of place - disaster - Québec 


\section{INTRODUCTION}

Le 6 juillet 2013, le centre-ville de la municipalité ${ }^{1}$ de Lac-Mégantic a été gravement atteint à la suite d'un important déraillement de train qui transportait du pétrole brut. Le feu et les explosions ont provoqué la destruction de 44 bâtiments et la contamination de 39 autres, ainsi que le confinement d'un large périmètre comprenant rues, parc, marina et accès à un lac renommé (figure 1). Cette destruction a amplifié un événement déjà tragique, puisque l'accident ferroviaire a entraîné le décès de 47 personnes. Après une phase d'urgence, une étape de décontamination a pris place, suivie de plusieurs décisions quant à la manière dont le centre-ville serait réhabilité, impliquant la démolition de bâtiments à proximité du centre-ville (quartier Fatima), mais surtout, la démolition des rues et des bâtiments encore debout dans le périmètre touché directement par le déversement d'hydrocarbures. La perte de ces traces, qui témoignaient du passé avant la tragédie, a créé une commotion chez les citoyens. Certains décrivent cette démolition comme une seconde tragédie, à la fois psychologique et sociale, laissant une blessure émotionnelle chez plusieurs citoyens de la région, ainsi qu'une déchirure dans la vie sociale et communautaire. Les traces laissées par le feu et les activités de décontamination sont également bien visibles, obligeant les résidents de la région méganticoise à faire un deuil du passé et de leur histoire et à s'adapter à un tout nouvel environnement.

Quoique singulière, cette situation n'est pas inusitée. Associée notamment à des pertes personnelles et à une diminution de la qualité de vie, la disparition d'un territoire significatif à la suite d'un déversement d'hydrocarbures est connue et elle engendre des impacts psychologiques comme le stress, l'anxiété, la dépression et le choc posttraumatique (Gill et Ritchie, 2015; Ritchie et al., 2012; Lee et Blanchard, 2012). Surtout, les étapes de décontamination et de reconstruction soulèvent des questions et des controverses auxquelles cette perte se conjugue, par exemple quant aux risques liés à l'environnement et quant à la gestion. À LacMégantic, des revendications concernant la destruction des bâtiments historiques contaminés, qui témoignaient de la fondation de la ville et représen-

1. Au Québec, la municipalité est l'entité détenant le gouvernement local, équivalent à la commune en France. taient pour certains les racines de la communauté, ont été soulevées. La création de nouveaux pôles commerciaux (immeubles commerciaux, secteur Fatima et secteur Laval) éparpillés dans la ville pour pallier la perte d'accès à la rue principale de la ville semble également lourde de conséquences pour les citoyens, autant au niveau de l'offre de services qu'au niveau de la vie sociale et communautaire. Après un bouleversement, que deviennent les liens d'attachement au lieu lorsque celui-ci est si violemment atteint par une catastrophe et par ses réaménagements? Comment l'action publique locale gère une catastrophe et la reconstruction des lieux, et dans quelle mesure peut-elle se préoccuper des liens identitaires, fonctionnels, sociaux et environnementaux avec le territoire? Ces questions nous paraissent cruciales dans un contexte de reconstruction post-catastrophe, puisqu'elles sont susceptibles d'influencer les opinions quant au risque et à la gestion du territoire.

Dans une approche ethnographique et à partir des résultats d'observations et de 57 entretiens semidirigés réalisés en 2014 et 2015 auprès de citoyens et d'acteurs socioéconomiques dans la région de Lac-Mégantic, nous cherchons à rendre compte de la manière dont le lien d'attachement au lieu peut être mobilisé, autant par les décideurs que par les citoyens, pour faire valoir des enjeux territoriaux variés. Ce faisant, l'article souhaite offrir une contribution originale à l'étude de l'anthropologie des catastrophes et vise à explorer la manière dont un « gouvernement des catastrophes » (Revet et Langumier, 2013) peut intégrer les dimensions identitaires et symboliques des lieux. La perspective choisie interroge la réponse des autorités à une situation catastrophique, en contexte de reconstruction. Ce choix semble particulièrement intéressant pour réfléchir à l'identité du lieu, à son évolution et au partage des rôles de la reconstruction. Après une présentation des notions associées à l'attachement au lieu, nous présenterons la méthodologie utilisée pour l'enquête, les résultats et l'analyse de ceux-ci. Nous décrirons d'abord des lieux significatifs, puis nous nous attarderons sur la manière dont ils sont mobilisés dans le processus de gestion et plus précisément lors de la reconstruction. 


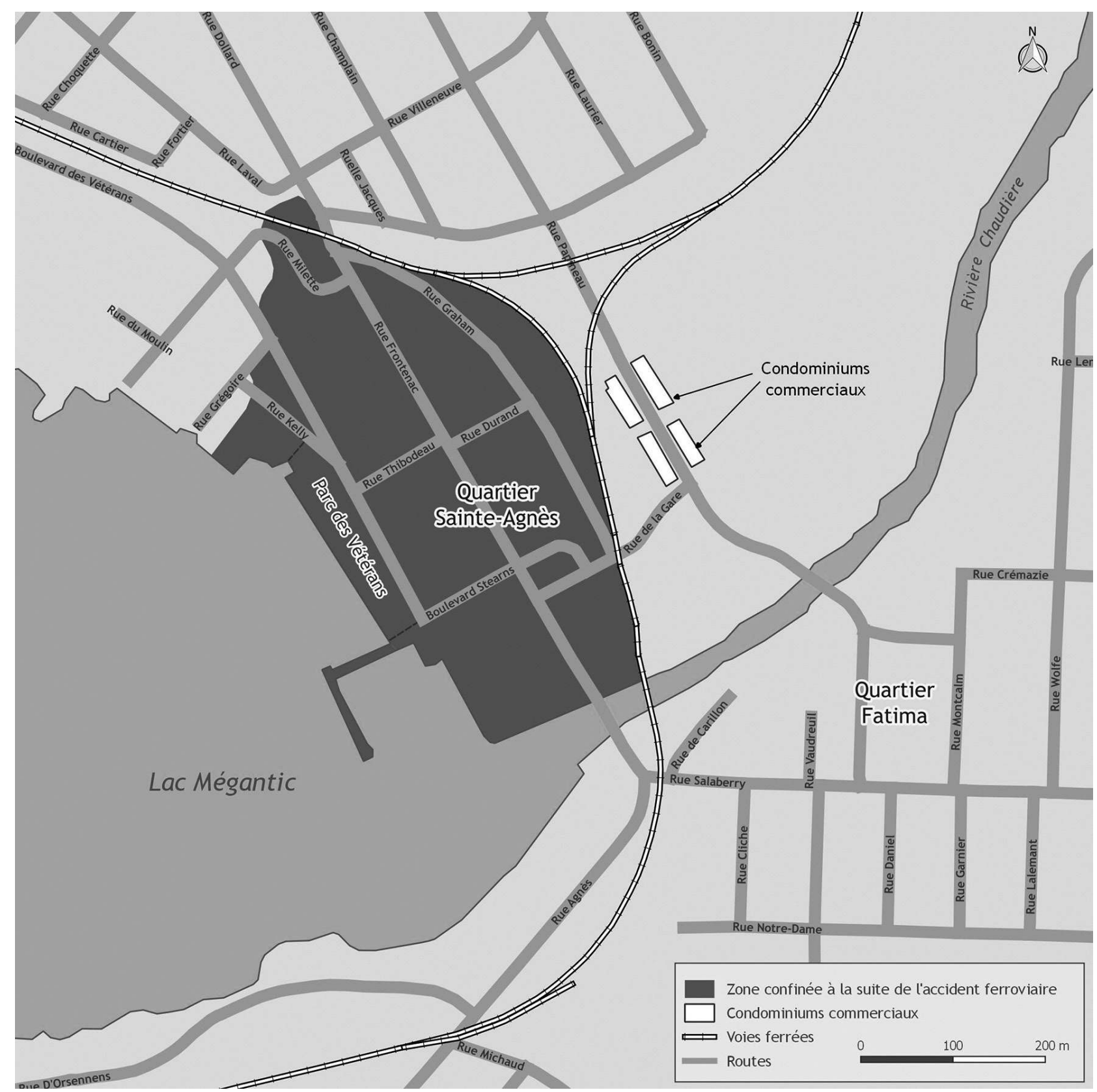

Figure 1 : Carte de localisation de la Ville de Lac-Mégantic après le déraillement (Réalisation : Matthieu Tandonnet, BIESP, INSPQ, 2018) Map of the Town of Lac-Mégantic after the derailment

\section{DES DYNAMIQUES SOCIOPOLITIQUES SPATIALISÉES}

Tout comme dans d'autres études sociales sur des catastrophes (Barrios, 2017 ; Button et Schuller, 2016; Revet et Langumier, 2013; Oliver-Smith et Hoffman, 1999), le processus de Lac-Mégantic met au jour des enjeux territoriaux intriqués, dont l'amé- nagement, les politiques publiques, les dynamiques d'acteurs, le rapport à la nature, l'identité et la culture. Ancrée dans l'écologie politique, notre étude souhaite d'abord mettre en lumière les dynamiques entre les acteurs en situation de gestion et durant le processus de reconstruction à court terme. Ces interactions rendent nécessaire l'analyse des relations de pouvoir, notamment celles issues du système éco- 
nomique et celles issues de la production du savoir et des discours (Bryant et Bailey, 1997). Dans le cas de Lac-Mégantic, les plans d'aménagement, les rencontres citoyennes, les décisions et les souhaits exprimés par les différentes parties prenantes seront au cœur de l'analyse (Brisson et Bouchard-Bastien, 2017 et 2016; Bouchard-Bastien et Brisson, 2016). Ces manifestations se lisent et agissent sur le milieu, contribuant à définir et à façonner les territoires tout autant que la compréhension des problématiques. L'action municipale s'incarne ici dans la capacité de nommer les choses, d'influencer les décisions et de produire des connaissances et des actions sur un milieu (Gauthier et Benjaminsen, 2012; Escobar, 1999), et elle s'inscrit dans des contextes sociaux, économiques, politiques et historiques qui modulent les interactions entre les humains et leur environnement. Ces contextes doivent être bien cernés pour mettre en lumière le lien d'attachement au lieu des Méganticois avec leur centre-ville (Escobar, 1999; Descola et Pàlson, 1996; Bryant, 1992).

La dimension spatiale où se jouent ces relations est centrale pour répondre aux questions de cet article, car elle rend lisible les dynamiques et les processus de pouvoir (November et al., 2004). Le sens des lieux (Basso et Feld, 1996) semble prégnant dans les discours et les actions mises en avant par les différents acteurs associés au processus de réhabilitation du centre-ville de Lac-Mégantic. Cette notion nous permet de réfléchir autrement à des processus d'aménagement du territoire, et notamment d'intégrer aussi bien des dimensions symboliques que politiques. Cette intégration nous semble nécessaire pour s'éloigner quelque peu des aspects psychosociaux de la tragédie, tout en mettant de l'avant des dimensions sensibles et expérientielles importantes pour l'anthropologie (Barrios, 2017).

\section{Le lien d'attachement au lieu}

Explorer en quoi l'attachement au lieu peut constituer un point focal dans la gestion de la situation permettra ici de constater la convergence de ces enjeux, et ce, à des échelles variées. L'attachement au lieu est souvent associé à la psychologie sociale de l'environnement, et le concept réfère alors aux théories psychologiques de l'attachement. Le terme est aussi utilisé par d'autres disciplines telles que la géographie, la sociologie, l'anthropologie et l'archi- tecture. La littérature sur le lien d'attachement au lieu propose plusieurs définitions théoriques pour identifier et comprendre sa nature. Malgré l'absence de consensus dans les définitions et l'abondance de la littérature sur le sujet, deux notions sont mises de l'avant par bon nombre d'auteurs comme composantes de ce lien, soit l'identité au lieu («place identity ») et la fonctionnalité du lieu («place dépendance»). Pour Proshansky (1978) et d'autres psychologues de l'environnement travaillant sur le logement, la collectivité, la région (Smith, 1994 ; Cuba et Hummon, 1993), la nature et le loisir (Williams, 2002), le lieu fait partie intégrante de l'identité, c'est-à-dire qu'il participe à la composition du Soi. La fonctionnalité du lieu, pour sa part, postule que l'attachement se base sur le lien qui permet d'établir que ce lieu est le plus adéquat pour réaliser une activité ou un intérêt spécifique (Brown et al., 2015; Raymond et al., 2010; Antonsich, 2010; Trentelman, 2009).

Ces deux notions font référence à un lien d'attachement au lieu basé sur une relation essentiellement cognitive, affective et comportementale, ce qui nous apparaît incomplet pour qualifier les dynamiques de gestion territoriale et prendre en compte les dimensions culturelles et symboliques de la reconstruction (Jorgensen et Stedman, 2002). De ce fait, des recherches plus récentes proposent d'élargir le concept et d'inclure des dimensions sociales et physiques dans la construction de l'attachement au lieu (Raymond et al., 2010; Antonsich, 2010). En réponse à cette nouvelle orientation, des auteurs ont développé d'autres modèles pour expliquer la construction de la notion du sens des lieux. À l'instar de Relph (1976), qui attribuait trois pôles («the physical setting, the activities and the meanings ") au concept d'identité au lieu, le sociologue Per Gustafson a proposé un modèle basé sur le Soi, les Autres et l'Environnement, qui permet de sortir de la dimension personnelle du lien d'attachement au lieu en intégrant de nouvelles dimensions (Turner et Turner, 2006; Gustafson, 2001). Plus récemment, Raymond et al. (2010) ont repris l'idée des trois pôles et ont élaboré une version pragmatique du modèle de Gustafson (Raymond et al., 2010; Turner et Turner, 2006) en reprenant les concepts d'identité, de fonctionnalité et d'attachement au lieu présents dans la littérature existante, en plus d'ajouter des notions telles que l'enracinement, 
l'appartenance, le lien avec la nature et l'implication des acteurs (leurs connaissances, leurs intérêts et leurs ressources). Cette approche de Raymond et al. (2010) sera retenue comme cadre analytique pour prendre en compte la variabilité des données ethnographiques collectées à Lac-Mégantic qui sont associées au lien d'attachement au lieu.

\section{Gouverner les catastrophes : le pouvoir sur les lieux}

La littérature scientifique démontre que les catastrophes peuvent modifier le contexte socioculturel et la perception de la communauté touchée, ce qui oblige cette dernière à reconstruire son univers symbolique et ses liens d'attachement au lieu (Brisson et Bouchard-Bastien, 2017 ; Ullberg, 2013; Enarson, 2000). Forcément, les pouvoirs locaux sont impliqués dans cette reconstruction (Barrios, 2017), et ce volet est au cœur de notre article.

En effet, comme le soulignent Revet et Langumier (2013), les catastrophes se gouvernent, ce sont " des événements aux impacts exceptionnels qui appellent une réponse » intense des autorités (Revet et Langumier, 2013) qui doivent agir et régler le bouleversement. Analysant de manière critique et sociopolitique ces actions, la « gouvernance des catastrophes » met au jour les dynamiques des interactions qu'impose une catastrophe et amène à s'intéresser aux acteurs locaux et aux autorités pour comprendre leurs différentes visions du monde, leurs expériences et leurs expertises. Cette approche permet aussi de voir comment ces éléments entrent en relation avec les sens des lieux passé et actuel, les affects et les contextes locaux, tels que représentés par les quatre dimensions (l'identité, la fonctionnalité, le social et la nature) de l'attachement au lieu de Raymond et al. (2010). Les autorités doivent généralement intervenir en urgence dans leur prise de décision, ce qui peut engendrer un décalage entre les règles de l'art et la pratique, tel que ce fut observé à Lac-Mégantic (Valiquette L'Heureux, 2016).

Puisque la réponse des autorités devient un moteur important (sinon principal) de la construction du lien d'attachement au nouveau lieu tout comme les capacités d'impliquer des acteurs (leurs connaissances, leurs intérêts et leurs ressources) (Mehnen et collab., 2013), l'article se penchera sur les enjeux de la reconstruction dans un contexte post-catastrophe et analysera les capacités locales de rétablir un milieu de vie et des lieux significatifs.

\section{Approche ethnographiQue DE LA QUESTION}

Cet article relate des résultats issus d'une recherche mandatée par la Direction de santé publique de l'Estrie. Cette dernière était préoccupée par les perceptions du risque et de la gestion, de même que par les effets psychologiques et sociaux persistants après la tragédie ${ }^{2}$, dans un contexte où les citoyens de Lac-Mégantic et des environs devaient composer avec le deuil des personnes disparues, la décontamination du site et la reconstruction du centre-ville, ainsi qu'avec les différents enjeux qui en découlent ${ }^{3}$.

Afin de capter l'influence des bouleversements successifs de la gestion post-catastrophe, deux phases de recrutement et d'entrevues ont été menées, soit en octobre-novembre 2014, et en janvier-février 2015. Au total, 57 entrevues ont été menées au sein de la population de la grande région de Lac-Mégantic. Un échantillon par quotas a été constitué (Gauthier et al., 2004) afin d'assurer une représentativité la plus fidèle possible de la population, et s'est composé de citoyens évacués $(\mathrm{n}=11)$, de citoyens des autres zones de LacMégantic $(\mathrm{n}=10)$ et de municipalités avoisinantes de Lac-Mégantic ( $\mathrm{n}=9)$; ainsi que d'acteurs socioéconomiques locaux (gens d'affaires, gestionnaires et professionnels gouvernementaux, analystes et coordonnateurs d'organismes communautaires, etc.) $(\mathrm{n}=27)$. La collecte des données a aussi comporté des séances d'observation réalisées lors de plusieurs assemblées d'information et des séances de consultation citoyenne dans le cadre d'une démarche consultative entreprise par les autorités locales et nommée « Réinventer la ville». Une analyse de contenu a permis de mettre au jour les dimensions

\footnotetext{
2. Intitulée «Préoccupations, opinions, apprentissageset souhaits quant aux risques et à la gestion des risques de la population de la région de LacMégantic ", le mandat de cette recherche à caractère ethnographique a été accordé à l'Institut national de santé publique du Québec (INSPQ) en décembre 2013.

3. Un certificat du comité d'éthique de la recherche de l'Université Laval a été obtenu pour ce projet (2014-192 / 20-10-2014). Cette procédure assure que les recherches portant sur des êtres humains se fassent dans le respect des personnes, et avec une préoccupation pour le bien-être et la justice.
} 
du modèle de Raymond et de ses collaborateurs (2010).

Parmi les limites de l'étude, il faut mentionner qu'il est possible que certains types de citoyens aient choisi de ne pas participer à l'étude, notamment pour se préserver au plan psychologique. Par ailleurs, l'étude reflète l'état de la situation entre 2014 et 2015 seulement. Le processus de rétablissement est dynamique et plusieurs changements ont eu lieu depuis la réalisation du travail de terrain à LacMégantic, notamment au sein du gouvernement municipal (élections) et dans l'avancée des travaux de reconstruction. Finalement, précisons que les lieux significatifs identifiés par les répondants l'ont été suite à l'accident. De ce fait, le sentiment d'attachement au lieu peut avoir été influencé par la tragédie, qui aurait détruit ou menacé certains lieux.

\section{DES LIEUX SIGNIFICATIFS À LAC-MÉgantic}

Cet article pose comme hypothèse que des lieux significatifs sont au cœur des enjeux du processus de gestion de la catastrophe et de la reconstruction à Lac-Mégantic. Mis au jour par les entrevues, ces lieux semblent se regrouper en quatre groupes, soit : les bâtiments historiques, l'environnement naturel, la rue Frontenac et la voie ferrée. La richesse des témoignages des participants et des observations sur le terrain permet d'associer ces lieux avec les quatre dimensions retenues qui entrent dans la composition du lien d'attachement au lieu.

\section{Des bâtiments représentatifs d'une époque}

La municipalité de Lac-Mégantic fut fondée en 1907 à la suite de la fusion de deux municipalités distinctes, soit les villages de Mégantic (paroisse Notre-Dame-de-Fatima) et d'Agnès (paroisse SainteAgnès), respectivement érigées en 1885 et 1895 (ville de Lac-Mégantic, 2012). Malgré cette fusion plus que centenaire, la différence entre les deux quartiers fondateurs demeure grâce à la séparation physique occasionnée par la rivière Chaudière, ainsi que par le type d'utilisation du territoire. Avant la tragédie, la majorité des services et des commerces se retrouvait dans le quartier Sainte-Agnès, alors que le quartier Fatima hébergeait les industries et les manufactures, ce qui en faisait un quartier à caractère ouvrier et offrant des habitations à prix modiques.

L'accident ferroviaire a détruit par le feu des lieux décrits comme étant des témoins du passé et entretenant l'identité méganticoise, soit une quarantaine de bâtiments (commerces et résidences) du secteur Sainte-Agnès. Le déversement d'hydrocarbures, pour sa part, a contaminé une zone relativement équivalente en termes de superficie à celle détruite par le feu. Cette zone a d'ailleurs été clôturée pendant les travaux de décontamination. Toutefois, c'est seulement en octobre 2014, soit plus d'un an après l'accident, que la municipalité a annoncé officiellement la destruction de ces bâtiments historiques encore debout dans la zone sinistrée (figure 2). Ce délai a été présenté lors des entrevues comme «un deuxième deuil ». Déjà affectés par la perte de leurs points de repère, les Méganticois ont espéré avoir à nouveau accès à ces bâtiments encore debout une fois les activités de décontamination terminées.

La perte de ces bâtiments a été associée à un déracinement, comme l'explique ce participant : «Ceux qui viennent d'ici, travaillent ici, c'est comme s'ils détruisaient le fondement de sur quoi se sont basées toutes leurs vies ». Ainsi, lors de la collecte des données, plus de la moitié des répondants ont fait état de leur attachement envers les bâtiments historiques détruits. Particulièrement lors de l'annonce de la destruction des bâtiments contaminés, plusieurs personnes ont manifesté le souhait de conserver des façades de bâtiments lors de la reconstruction. Ce souhait ne s'est finalement pas réalisé, et la

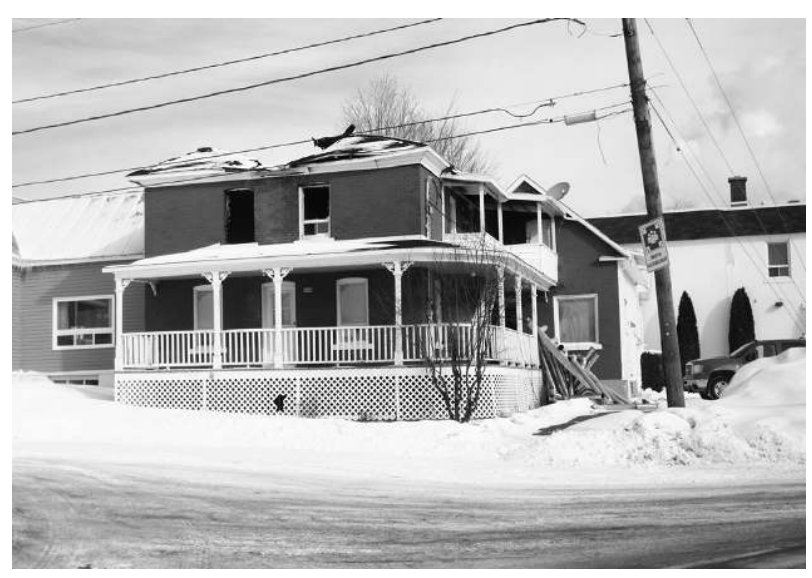

Figure 2 : Bâtiments historiques aujourd'hui détruits (Source : Emmanuelle Bouchard-Bastien, janvier 2014) Historic buildings now destroyed 
nouvelle architecture de style épuré mise en avant par la Ville a été critiquée par la majorité des participants rencontrés (ce fait sera explicité dans la partie suivante). Le soir de l'annonce de la destruction des bâtiments, une demande a également été formulée à la Ville par une citoyenne, consistant à pouvoir circuler une dernière fois dans la zone contaminée avant sa destruction. C'est ainsi que « La dernière marche » fut organisée par la municipalité le 6 décembre 2014, ce qui permit aux résidents de la région d'avoir un dernier contact avec ce lieu historique chargé de souvenirs tristes et heureux.

\section{Une nature familière et réconfortante}

Comme son nom l'indique, la municipalité de LacMégantic est située sur les berges du lac Mégantic, qui constitue la tête de la rivière Chaudière, et qui se décharge 185 kilomètres plus loin dans le fleuve Saint-Laurent à la hauteur de la ville de Québec (idem). Les parcs nationaux du Mont-Mégantic et de Frontenac sont situés à proximité, ainsi que l'Observatoire d'astronomie le plus puissant du Canada (SÉPAQ, 2016). De ce fait, la région de LacMégantic se situe au coeur d'une réserve internationale de ciel étoilé, statut qui assure la lutte contre la pollution lumineuse dans la région afin de conserver l'accès à l'observation des étoiles (RICEMM, s.d.).

Que ce soit parce qu'ils évoluent dans un milieu où la nature est omniprésente, ou parce qu'ils ont été victimes d'une catastrophe environnementale, les participants à l'étude ont été très loquaces sur leur attachement à l'environnement naturel, particulièrement envers la rivière Chaudière, le lac Mégantic et le parc des Vétérans, un parc en plein centre-ville, au bord du lac Mégantic et possédant de majestueux arbres centenaires. Ne plus avoir accès à l'eau (ou à certaines activités telles que la pêche et la baignade) et au parc des Vétérans suite à l'accident et pendant la période de décontamination a été vécu par certains informateurs comme une diminution de la qualité de vie, puisque c'était " un lieu d'aération de l'esprit », où il était possible de «décompresser». De ce fait, un bon nombre d'informateurs entretenait un lien de familiarité avec l'environnement naturel, c'est-à-dire, une grande intimité née d'un rapport constant avec la nature.

Par ailleurs, le déversement d'hydrocarbure engendré par l'accident ferroviaire a donné lieu à d'impor- tants travaux de décontamination qui ont duré 2 ans et 4 mois. Quotidiennement, les Méganticois ont été témoin du pompage d'eau souillée aux hydrocarbures et du déplacement par camions de la terre contaminée de la zone sinistrée vers la zone industrielle, en plus des équipements qui assuraient la surveillance de la qualité de l'air et de l'eau de baignade. Des craintes pour la régénération de la nature au centre-ville ont été vécues durant cette période par certains habitants, comme le souligne ce citadin natif de Lac-Mégantic : «...quand tout va être nettoyé, que les bâtiments vont avoir été enlevés, qu'ils auront mis du sable partout, mais est-ce que c'est la nature ça?».

\section{Une rue principale rassembleuse}

La rue Frontenac était la rue principale de la ville de Lac-Mégantic. Parsemée des principaux commerces et services de la municipalité, tels qu'une épicerie, une pharmacie, un nettoyeur et une buanderie, une banque, un bar laitier et la bibliothèque, cette rue était un pôle de services très fréquenté. Suite à l'accident ferroviaire, la perte d'accès à ces services a ébranlé le lien d'attachement fonctionnel au lieu, autant chez les consommateurs que chez les commerçants. Pendant plusieurs mois, une baisse de l'offre de service a été vécue par la population de la région, comme en témoigne cette résidente, qui explique que lorsqu'elle a besoin de quelque chose à Lac-Mégantic, « $90 \%$ du temps, tu ne l'as pas ». Cette situation fut particulièrement problématique pour certains résidents du centre-ville qui ne possédaient pas de voiture, puisque des commerces offrant des biens essentiels, tels que l'épicerie et la pharmacie, s'étaient relocalisés temporairement dans le secteur Laval-Nord à plus de 4 kilomètres de leur endroit initial.

En raison de son fort achalandage, la rue Frontenac était également un haut lieu de la vie communautaire et sociale. Animé de jour comme de nuit, ce lieu permettait la rencontre informelle entre les membres de la communauté (le voisinage), comme l'explique ce résident du quartier Fatima : "On allait prendre une marche, on voyait toujours quelqu'un qu'on connaissait ». Un bon nombre de participants ont évoqué avec nostalgie les promenades sur la rue Frontenac après le souper, en mangeant une crème glacée. D'autres ont expliqué ne 
plus aller aux restaurants ou prendre un verre avec les amis, par manque d'endroits pour le faire. Les Méganticois ont donc perdu avec la rue Frontenac des lieux de rencontre, ce qui affecta considérablement le capital social de certains résidents.

\section{La voie ferrée : entre prospérité économique et souvenir douloureux}

Le dernier lieu significatif identifié par un grand nombre de participants est la voie ferrée. La construction de la voie ferrée a joué un rôle fondamental dans le développement de la région de Lac-Mégantic. En effet, la région est devenue un pôle ferroviaire stratégique dans la province grâce au passage des deux tracés en importance, soit le Canadien Pacifique (CP) et le Québec Central Railway (QCR) (MCC, 2013). Encore aujourd'hui, plusieurs emplois dépendent de la voie ferrée, et la municipalité de Lac-Mégantic est devenue avec le temps un centre ferroviaire régional orienté à la fois vers la Beauce, l'Estrie et l'état américain du Maine (Ville de Lac-Mégantic, 2012). Le patrimoine ferroviaire de Lac-Mégantic est donc associé à un fort sentiment d'appartenance pour la population. Toutefois, depuis l'accident ferroviaire, la perception du train a changé, puisqu'il est maintenant considéré comme une source de danger (Brisson et Bouchard-Bastien, 2017). Un bon nombre de participants craignent maintenant le transport ferroviaire de matières dangereuses. Toutefois, peu d'entre eux nient l'importance des enjeux économiques régionaux associés à la voie ferrée, comme l'explique cette participante : «Tout le monde est contre le passage du pétrole, mais on sait qu'on a besoin de ce chemin de fer là pour faire virer l'économie ici. On vit quand même en région... » De ce fait, des pourparlers concernant une voie de contournement du chemin de fer à l'extérieur du centre-ville sont en cours. Cette alternative semble être le meilleur moyen pour conserver la voie ferrée tout en atténuant les associations négatives qui en émanent.

\section{De multiples dimensions pour l'attachement au lieu}

Le modèle de Raymond et al. (2010) propose quatre dimensions qui entrent dans la composition de l'attachement au lieu, soit le lien identitaire, le lien fonctionnel, le lien social et le lien avec la nature. Ces dimensions théoriques du sens des lieux se lisent à Lac-Mégantic (tableau 1). Ainsi, de par son histoire et son style architectural évocateur du passé, les bâtiments du centre-ville nouent un lien d'attachement au lieu basé sur l'identité et l'enracinement. L'environnement naturel entourant le centre-ville de Lac-Mégantic évoque pour sa part l'importance de la nature sauvage et la familiarité, tandis que les témoignages des informateurs permettent d'associer les dimensions de la fonctionnalité et du voisinage aux liens qui relient les participants à la rue Frontenac. Enfin, à la lumière des données recueillies, la voie ferrée est associée à la fois à l'importance du patrimoine (sentiment d'appartenance) et à son importance économique (fonctionnel).

\begin{tabular}{|c|c|}
\hline $\begin{array}{c}\text { Lieux évoquant un lien } \\
\text { d'attachement }\end{array}$ & $\begin{array}{c}\text { Dimensions du sens } \\
\text { des lieux }\end{array}$ \\
\hline Bâtiments historiques & $\begin{array}{c}\text { Identité } \\
\text { Sociale (Enracinement) }\end{array}$ \\
\hline $\begin{array}{c}\text { Environnement naturel (lac } \\
\text { Mégantic, rivière Chaudière } \\
\text { et parc des Vétérans) }\end{array}$ & $\begin{array}{c}\text { Sociale (Familiarité) } \\
\text { Nature }\end{array}$ \\
\hline Rue Frontenac & $\begin{array}{c}\text { Fonctionnel } \\
\text { Sociale (Voisinage) }\end{array}$ \\
\hline Voie ferrée & $\begin{array}{c}\text { Fonctionnel } \\
\text { Sociale (Appartenance) }\end{array}$ \\
\hline
\end{tabular}

Tableau 1 : Les lieux évoquant un lien d'attachement à Lac-Mégantic

Places evoking a bond of attachment to Lac-Mégantic

\section{DES LIEUX MOBILISÉS? LA RECONSTRUCTION DES LIEUX PAR LA MUNICIPALITÉ}

Mais ces premiers résultats ne racontent pas toute l'histoire, puisqu'il s'agit maintenant de constater si ces lieux significatifs ont été (ou non) pris en considération lors de la reconstruction. Peu de temps après la tragédie, la Ville de Lac-Mégantic a adopté deux modalités afin d'orienter ses actions de reconstruction du centre-ville. D'une part un programme particulier d'urbanisme (PPU) (Ville de Lac-Mégantic, 2013), et d'autre part une démarche de consultation publique intitulée « Réinventer la ville ». 
Constatant que la zone sinistrée serait obligatoirement inoccupée pendant les travaux de décontamination, le PPU fut adopté afin de répondre au besoin de relocalisation des " commerçants majeurs cherch[a]nt des sites pour se reconstruire de manière permanente » (Ville de Lac-Mégantic, 2013). Du même souffle, le PPU avance que le « quartier Fatima qui, historiquement, a joué un rôle déterminant dans la fondation de la Ville de Lac-Mégantic » présente aujourd'hui « des signes alarmants de dévitalisation économique », alors qu’il recèle « des atouts importants en termes de localisation stratégique $[\ldots]$ et en matière de disponibilités d'immeubles pour un redéveloppement urbain potentiel » (idem). C'est ainsi que des expropriations dans le secteur de Fatima ont été autorisées à la suite de l'accident, afin de faire place à la pharmacie et des grandes surfaces d'alimentation. Cette décision plus ou moins bien acceptée par la population s'accompagne de nouvelles destructions de bâtiments historiques. Elle a contraint des citoyens du quartier Fatima à devoir se détacher de leur quartier, ce qui a modifié la trame sociale du quartier, comme l'explique ce résident : «La Ville a détruit le tissu social de Fatima ». Dans cette optique de devoir relocaliser des commerçants sinistrés, la Ville de Lac-Mégantic a également construit dès l'automne 2013 des condominiums (locaux) commerciaux sur un terrain lui appartenant (figure 3). Malgré son souhait de créer un lieu incontournable avec une expérience de magasinage conviviale (Ville de Lac-Mégantic, 2013), la majorité des participants rencontrés trouve plutôt que cet espace n'a pas su recréer la vie sociale et communautaire d'antan, entre autres du fait qu'il ne soit pas habité. Selon les personnes rencontrées, l'architecture « carrée, grise, laide » de ces bâtiments utilitaires et à vocation initialement temporaire contribue également au sentiment de «froideur » qui émane de l'endroit.

Dans un autre ordre d'idées, en mars 2014, la Ville de Lac-Mégantic a mis en place une démarche de participation citoyenne nommée "Réinventer la ville », afin de " définir collectivement le plan de reconstruction du centre-ville » (Ville de LacMégantic, 2014 :6). Cette démarche innovante et prometteuse s'est avérée toutefois un échec. Un bon nombre de participants rencontrés ont été emballés par la démarche lors de son inauguration, mais après quelques assemblées publiques et ateliers commu-

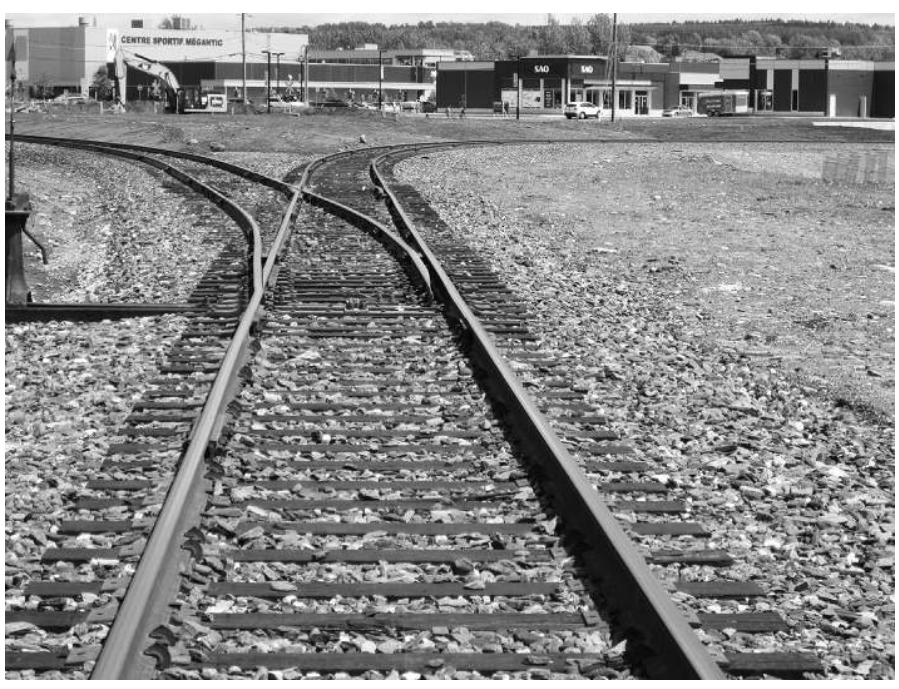

Figure 3 : La voie ferrée restaurée et les immeubles commerciaux (Source : Emmanuelle Bouchard-Bastien, juin 2014)

The restored railway and commercial condominiums

nautaires, ils ont réalisé que " tout était décidé d'avance » et «que les plans étaient déjà faits ${ }^{4}$ ». Comme en témoigne cette participante : «Des fois, on a l'impression de se faire prendre pour des nonos. C'est ça que, je crois, les gens n'acceptent pas. C'est ça qu'on entend autour. Ils nous font faire des tables rondes, mais leur décision est déjà prise. Ils vont peut-être retenir un petit point, pour dire « on a ajouté ça ». Mais réinventer? Non ». Le manque d'écoute de la part de la municipalité envers ses citoyens a été souligné à maintes reprises par les répondants, particulièrement concernant les décisions importantes. En effet, l'annonce de la destruction des bâtiments du centre-ville en octobre 2014 semble avoir donné le coup de grâce à la démarche, car la majorité des participants avait manifesté leur souhait de les conserver.

Il aura fallu quinze mois aux autorités municipales pour annoncer officiellement la destruction des bâtiments contaminés encore debout. Cette décision, prise conjointement avec le ministère de l'Environnement ${ }^{5}$, a permis de procéder à la décontamination complète de cet espace, et d'atteindre ainsi le risque zéro pour encourager les promoteurs et les commerçants à reconstruire dans la zone sinistrée. Cette préoccupation forte de la Ville était déjà présente

4. Le Plan directeur de reconstruction qui émane de la démarche « Réinventer la ville » propose les mêmes orientations prévues que le PPU.

5. Le nom complet du ministère du Développement durable, de l'Environnement et de la Lutte contre les Changements climatiques (MDDELCC) a été abrégé pour ne pas alourdir le texte. 
dans le PPU : « La Ville de Lac-Mégantic encourt un grand risque. Si la Ville ne trouve pas de solution à la relocalisation des entreprises qui œuvraient dans le centre-ville historique, les gens d'affaires auront tôt fait de se réinstaller hors du secteur central pour ne plus y revenir » (Ville de Lac-Mégantic, 2013).

Malgré la démolition de plus de 80 bâtiments commerciaux et résidentiels, le Plan directeur issu de «Réinventer la ville» souhaite explicitement entretenir un «lien entre le passé et l'avenir » du centre-ville (Ville de Lac-Mégantic, 2014). La préservation du tracé d'origine de la rue Frontenac et sa vocation de « rue principale » sont ainsi privilégiées, mais son aménagement et son apparence seront évidemment appelés à changer (idem). Selon les plans et les premiers projets officiels à ce jour, ce souhait sera toutefois difficile à réaliser pour la Ville. Certes, les nouveaux bâtiments adoptent résolument le style architectural épuré des immeubles commerciaux, mais cette nouvelle image de marque ne sera pas la plus grande différence entre le passé et l'avenir. Les délais importants occasionnés par le processus de décontamination et le rachat des bâtiments de la zone sinistrée par la Ville ${ }^{6}$, deux processus orchestrés en partenariat avec le gouvernement du Québec, ont obligé la majorité des commerçants à se relocaliser ailleurs pour éviter la faillite, en plus de décourager certains résidents à se reconstruire. La dispersion des commerces et des résidents du centre-ville laisse planer des craintes quant à la reprise de la vitalité économique et du retour de la vie sociale et communautaire sur la rue Frontenac.

En somme, les actions de la municipalité de Lac-Mégantic dans le cadre de la reconstruction semblent particulièrement orientées vers une vocation économique. Des préoccupations historiques, sociales et culturelles sont également identifiées dans les documents d'orientations, telles que la valorisation du patrimoine et la création de lieux conviviaux, mais ces dernières demeurent difficilement perceptibles dans les actions concrètes. À l'instar d'autres autorités ayant eu à prendre en charge des lieux sinistrés, les gestionnaires et les experts derrière le PPU et la démarche "Réinventer la ville » semblent reconstruire le centre-ville de Lac-

6. Les bâtiments contaminés encore debout après l'accident ferroviaire ont été rachetés par la Ville de Lac-Mégantic afin de procéder à la décontamination complète du site. Ce rachat a été possible grâce à une subvention de 60 millions \$CAN offerte par le ministère des Affaires municipales et de l'Occupation du territoire (MAMOT).
Mégantic en omettant de prendre en compte les significations ou les dynamiques sociales qui façonnaient le centre-ville (Barrios, 2017, Marchezini, 2015). Favoriser la prospérité économique et l’innovation technologique fait partie du mandat d'une organisation publique municipale, mais dans le contexte de reconstruction de Lac-Mégantic, ces actions s'avèrent désincarnées de la majorité des gens qui habitent et utilisent ce territoire.

\section{ANALYSE DE LA MOBILISATION DES LIEUX DANS LA GESTION MUNICIPALE}

Certaines données recueillies à Lac-Mégantic à la suite de l'accident ferroviaire ont permis d'identifier les dimensions du lien d'attachement au lieu associé au centre-ville et de mettre au jour les dynamiques intriquées des acteurs et des enjeux. Lors de la collecte de données, le processus de reconstruction n'était pas abouti, mais déjà, certaines réponses des autorités ne faisaient pas l'unanimité. Était-ce parce que les réponses des autorités ne véhiculaient pas les liens identitaires, fonctionnels, sociaux et environnementaux que les citoyens entretenaient avec le territoire du centre-ville?

Malgré le fait que des membres composant l'autorité municipale soient également des sinistrés, et que pour certains, la perte du centre-ville soit également associée à une perte significative, la préoccupation qui semble orienter la grande majorité des réponses des autorités est la relance économique, comme en témoigne son omniprésence dans le PPU. D'ailleurs, la rapidité avec laquelle ce règlement fut élaboré suite à l'accident amène certains citoyens à croire que la tragédie ferroviaire a été le prétexte permettant aux élus municipaux de réorienter l'économie de la ville vers le tourisme, conformément aux orientations pré-existantes à l'événement. Les préoccupations économiques étaient également bien présentes chez les Méganticois, mais elles s'inscrivaient plutôt dans la dimension fonctionnelle du lien d'attachement au lieu (Raymond et coll., 2010; Antonsich, 2010; Trentelman, 2009). Dans cette optique, elles visaient à assurer le maintien de l'offre de services de la rue Frontenac et des activités ferroviaires comme bénéfice économique, et non le développement de l'offre touristique. Le plan directeur, pour sa part, propose un centre-ville axé sur 
le tourisme avec de nouveaux pôles commerciaux qui risque de concurrencer l'offre commerciale de la future rue Frontenac. La réponse des autorités est donc animée par une préoccupation qui est chère aux Méganticois, mais elle propose une autre interprétation des lieux qui y sont associés pour arriver à la même finalité, qui est la prospérité économique. Cet exemple met en lumière que la façon dont la problématisation des enjeux de reconstruction qui a été réalisée n’a pas permis la prise en compte du lien d'attachement des Méganticois avec leur centreville. Réaménager le territoire demande de comprendre en profondeur les contextes sociaux, économiques, politiques et historiques qui modulent les interactions entre les humains et leur environnement (Escobar, 1999; Descola et Pàlson, 1996; Bryant, 1992). Dans ce cas-ci, l'enjeu de reconstruction était bien une question de prospérité économique, mais la réponse des autorités à cette préoccupation citoyenne, basée sur la dimension fonctionnelle au lieu, devait permettre aux Méganticois de poursuivre leurs activités et de réaliser leurs intérêts (Raymond et al., 2010; Antonsich, 2010 ; Trentelman, 2009). Or, les contraintes environnementales (décontamination) et financières (rachat des bâtiments) occasionnées par les partenariats avec les instances gouvernementales ont amené les autorités qui pilotaient la reconstruction à opter pour une réponse basée sur un discours économique désincarné de la relation citoyens-espace en vigueur, ce qui amène sans le vouloir une rupture dans cette relation qui assurait le bien-être de la communauté (Barrios, 2017; Bryant et Bailey, 1997). En ce sens, un conseiller municipal s'est senti «mis en tutelle » par le gouvernement du Québec et a déploré de devoir obtenir des préautorisations de la part des fonctionnaires gouvernementaux avant de pouvoir intervenir : «Ils ne viennent pas sur place et ne nous connaissent pas! Ça retarde! »

Le cas de la voie ferrée est également éloquent à cet égard, car le chemin de fer disparaît des plans du futur centre-ville, alors qu'elle fut la première infrastructure reconstruite suite au sinistre afin de ne pas entraver les activités d'exportation du plus gros employeur de la région ${ }^{7}$. Cette orientation semble donc décalée avec les considérations

7. L'usine Tafisa, qui est la plus grande usine de production de panneaux de particules en Amérique du Nord, est située à Lac-Mégantic et emploie 325 travailleurs. économiques en vigueur à Lac-Mégantic. Certes, il est important de préciser que la disparition de la voie ferrée dans les futurs plans du centre-ville concorde avec le souhait de plusieurs membres de cette communauté rudement éprouvée. Mais ce souhait n'est pas unanime, et la dimension de l'identité au lieu peut expliquer en partie pourquoi. La voie ferrée, tout comme les bâtiments historiques du centre-ville, s'inscrit de manière importante dans l'histoire de la région et agit dans la composition du lien d'attachement au lieu. Or, la réponse des autorités municipales n'amène aucun engagement concret à l'heure actuelle permettant de valoriser la poursuite des activités ferroviaires et de réhabiliter cette industrie. L'omission de la voie ferrée dans les plans du nouveau centre-ville amenuise le lien identitaire et fonctionnel entre Lac-Mégantic et cette industrie. Cette action des autorités fait d'ailleurs dire à un représentant gouvernemental que « La Ville rêve » et que projeter ces images aux citoyens représente un risque, puisque la Ville n'a ni la légitimité d'intervenir dans le domaine ferroviaire ni les moyens financiers de se priver d'une voie ferrée. La voie ferrée illustre la complexité des enjeux à laquelle les autorités peuvent être confrontées à la suite d'une catastrophe (Revet et Langumier, 2013). La reconstruction d'un univers symbolique intègre plusieurs dimensions qui modulent les liens d'attachement au lieu (Ullberg, 2013; Enarson, 2000). Pour certains citoyens de Lac-Mégantic, le train est dorénavant synonyme de danger et sa disparition du centre-ville devient un soulagement (Brisson et Bouchard-Bastien, 2017). Toutefois, la voie ferrée fait également partie de l'identité de la communauté, puisqu'elle en est un des éléments fondateurs de la ville, et sa disparition devient alors une perte d'identité et d'appartenance. Dans ce cas-ci, la gestion mise en place par les autorités municipales crée une tension entre le passé, l'urgence de la situation et les décisions concertées, et modifie forcément le lien d'attachement de la communauté à la voie ferrée (Mehen et al., 2013; Revet et Langumier, 2013).

La dimension de l'appartenance et du voisinage associée à la vie sociale et communautaire et au contact avec la nature est un autre élément important dans le lien d'attachement au lieu des Méganticois qui peine à se retrouver dans la réponse des autorités. Les nouveaux aménagements construits à la hâte à la suite de l'accident n'ont pas 
réussi à recréer un lieu propice aux rencontres. Les immeubles commerciaux sont éloignés de l'ancien centre-ville (et donc du bord de lac), présentent une architecture épurée qui rappelle sa vocation utilitaire, et se situe dans une zone exclusivement commerciale, ce qui rend les lieux complètement déserts après la fermeture des commerces. L'absence de concertation dans la conception de l'aménagement du territoire explique certainement en partie pourquoi la majorité des participants à l'étude craignent de se retrouver avec un centre-ville qui ne sera pas à leur image (Barrios, 2017; Valiquette L'Heureux, 2016). Le centre-ville touristique projeté, avant-gardiste et écologique, ne tient pas compte des habitudes de vies des citoyens qui fréquentaient jadis cette zone.

La seule dimension qui semble prise en compte dans la réponse des autorités pour la reconstruction du centre-ville est celle associée à la nature sauvage; elle fait partie de l'offre touristique. Ainsi, le maintien d'un accès visuel et physique au lac Mégantic grâce à la conservation intégrale du parc des Vétérans fait partie des priorités des élus, ainsi que l'aménagement d'un « Jardin des étoiles » pour valoriser le statut de réserve étoilée de la région (Ville de Lac-Mégantic, 2014 : 35). Le fait de ne pas intégrer la voie ferrée dans le plan directeur, et par le fait même, d'éloigner le transport de matière dangereuse du centre-ville, concorde également avec la préservation de la nature. Toutefois, l'offre touristique principalement orientée vers la valeur «écologique» ne permet pas de prendre en compte la dimension de la nature. Cette réponse demeure sous l'égide des valeurs économiques. Les projets immobiliers annoncés (marina, complexe hôtelier, centre de santé) qui proposent une architecture écologique amènent également une déception au niveau de la dimension identitaire de plusieurs résidents, car leur architecture épurée rappelle la froideur des condominiums commerciaux.

\section{Conclusion : GÉRER LA RECONS- TRUCTION ET TENIR COMPTE DES SIGNIFICATIONS}

À la suite de Gustafson (2001) et de Raymond et al. (2010), le concept du sens des lieux ainsi que les pôles et dimensions qui contribuent au lien d'attachement au lieu ont été utilisés dans le cadre de cette analyse. Cette démarche a permis d'évaluer, dans une perspective de gestion et d'aménagement du territoire, comment la réponse des autorités dans le cadre d'une reconstruction post-catastrophe permet de demeurer fidèle (ou non) aux significations spatiales attribuées par les citoyens de la région.

Les résultats de cette analyse montrent que des lieux significatifs sont au cœur des enjeux du processus de gestion de la catastrophe et de la reconstruction à Lac-Mégantic. Comme dans d'autres cas de reconstruction post-sinistre, cette illustration suggère que la réalisation d'un aménagement du territoire porteur de sens pour ses habitants ne peut se réaliser en huis clos, sur une carte vierge, sans tenir compte des dynamiques sociopolitiques, socioéconomiques et culturelles des citoyens (Barrios, 2017; Revet et Langumier, 2013). Plus particulièrement, les résultats de Lac-Mégantic mènent à penser que la construction d'un espace significatif a besoin d'être ancrée à la fois dans l'environnement concret et dans les relations sociales et personnelles; elle ne peut être seulement élaborée à partir d'une relation behavioriste et affective (Jorgensen et Stedman, 2002), ni uniquement à partir de schémas abstraits (Trentelman, 2009).

Les résultats démontrent également que la reconstruction de lieux significatifs représente un immense défi de gestion pour de petites autorités municipales telles que celle de Lac-Mégantic. Ce défi se définit aussi bien quant aux ressources humaines et financières disponibles que par rapport aux nombreuses décisions à prendre dans un court laps de temps. Dans ce cas précis, l'enjeu de la décontamination du centre-ville a été particulièrement complexe à gérer, puisqu'il a amené dans l'arène locale des acteurs provinciaux, des coûts supérieurs à la simple reconstruction de bâtiments et d'importants délais d'exécution. Ces considérations ont fortement modulé l'action publique locale concernant la prise en compte des liens d'attachement associés aux bâtiments historiques et à la rue Frontenac. Le déraillement de train a pour sa part modifié la perception d'un bon nombre de Méganticois envers la voie ferrée, et ce changement a également complexifié la réponse des autorités municipales, qui devaient tenir compte à la fois des liens fonctionnels et d'appartenance en vigueur et des impacts traumatiques générés par l'accident. 
En vertu de ses pouvoirs et de la proximité qu'il entretient avec les citoyens, le monde municipal (et local) est sans doute le mieux placé pour prendre en charge la reconstruction des lieux sinistrés, assurer une continuité dans les valeurs territoriales et créer de nouveaux lieux significatifs. Reconstruire des lieux significatifs s'avère toutefois une lourde tâche pour cette entité, qui peut être confrontée par une limitation de ses pouvoirs de la part d'autres paliers gouvernementaux ou encore par un rôle qui diffère de ses sphères d'action habituelles.

\section{Remerciements}

Les deux auteures souhaitent remercier tous les participants à cette étude, car sans eux, rien n'aurait été possible. Sincères remerciements aux membres du cercle d'écriture du Centre de recherche en développement territorial (CRDT) pour leurs commentaires riches et pertinents tout au long de la rédaction de cet article. Nous remercions également les deux évaluateurs anonymes qui ont permis de bonifier cet article grâce à leurs commentaires justes et constructifs. Cette recherche a été financée par l'Institut national de santé publique du Québec et la Direction de santé publique de l'Estrie.

\section{Bibliographie}

Antonsich M., 2010. Meanings of Place and Aspects of the Self : an Interdisciplinary and Empirical Account, GeoJournal, $\mathrm{n}^{0}$ 1, vol. 75, p. 119-132. DOI: 10.1007/s10708-0099290-9.

Barrios R.E., 2017. Governing Affect: Neoliberalism and Disaster Reconstruction, Lincoln/London, University of Nebraska Press, 288 p.

Basso K., Feld S., 1996. Senses of Place, School of American Research Press, Santa Fe, 293 p.

Bouchard-Bastien E. et Brisson G., 2016. Changements sociaux et risques perçus à la suite de la tragédie ferroviaire de Lac-Mégantic : rapport de recherche, Institut national de santé publique du Québec, Québec, 65 p. Consulté à l'adresse : [https://www.inspq.qc.ca/publications/2210].

Brisson G., Bouchard-Bastien E., 2017. With or Without Railway? Post-Catastrophe Perceptions of Risk and Development in Lac-Mégantic, Québec, in Kirk J., Willow A., Casagrande D., Paladino S. (dir.), ExtraCTiOn: Impacts, Engagements, and Alternative Futures, London/New York, Routledge, p. 123-136.
Brisson G., Bouchard-Bastien E., 2016. Opinions locales quant à la gestion des risques et du rétablissement à la suite de la tragédie ferroviaire de Lac-Mégantic : rapport de recherche, Institut national de santé publique du Québec, Québec, 67 p. Consulté à l'adresse : [https://www.inspq.qc.ca/publications/2211]

Brown G., Raymond C.M., Corcoran J., 2015. Mapping and Measuring Place Attachement, Applied Geography, no 57, p. 42-53. DOI: 10.1016/j.apgeog.2014.12.011.

Bryant R., Bailey S., 1997. A Politicised Environment, in Bryant R., Bailey S., Third world political ecology, London/ New York, Routledge, p. 26-45.

Bryant R., 1992. Political Ecology: An Emerging Research Agenda in Third-Wolrd Studies, Political Geography, n ${ }^{\circ}$, vol. 11, p. 12-36. DOI: 10.1016/0962-6298(92)90017-N.

Button B.V., Schuller M., 2016. Contextualising Disaster, New York, Berghahn Books, 208 p.

Cuba L., Hummon D.M., 1993. A place to call home: Identification with dwelling, community, and region, The Sociological Quarterly, no 1, vol. 34, p. 111-131. DOI: $10.1111 / \mathrm{j}$. 1533-8525.1993.tb00133.x.

Descola P., Pàlsson G., 1996. Nature ans Society. Anthropological Perspectives, New York, Routledge, $310 \mathrm{p}$.

Enarson E., 2000. "We Will Make Meaning Out of This": Women's Cultural Responses to the Red River Valley Flood. International Journal of Mass Emergencies and Disasters, $\mathrm{n}^{\circ} 1$, vol. 18 , p. 39-62.

Escobar A., 1999. After Nature Steps to an Antiessentialist Political Ecology, Annual Reviews of Anthropology, $\mathrm{n}^{\circ}$ 1, vol. 40, p. 1-30. DOI: 10.1086/515799.

Gauthier D., Benjaminsen T.A., 2012. Environnement, discours et pouvoir : l'approche political ecology, Versailles, Quae, coll. «Update sciences \& technologies », 256 p.

Gauthier B. (dir.), 2004. Recherche sociale. De la problématique à la collecte de données, Presses de l'Université du Québec, Québec, 619 p.

Gill D.A., Ritchie L.A., 2015. Sociocultural Survival in the Face of Looming Disaster: the Gitga'at First nation's Opposition to the Enbridge Northern Gateway Pipeline project, in Companion M. (dir.). Disaster's Impact on Livelihood and Cultural Survival: Losses, Opportunities, and Mitigation. Éditions CRC Press, p. 211-224.

Gustafson P., 2001. Meanings of Place : Everyday Experience and Theorical Conceptualisations, Journal of Environmental Psychology, n 1, vol. 21, p. 5-16. DOI: 10.1006/ jevp.2000.0185.

Jorgensen B.S., Stedman R.C., 2001. Sense of Place as an Attitude: Lakeshore Owners Attitudes towards their Properties, Journal of Environmental Psychology, $\mathrm{n}^{\circ}$ 1, vol. 21, p. 233-248. DOI: 10.1006/jevp.2001.0226.

Lee M.R., Blanchard T.C., 2012. Community attachment and negative affective states in the context of the BP Deepwater Horizon disaster. American Behavioral Scientist, $\mathrm{n}^{0} 56$, vol. 1, p. 24-47. DOI: 10.1177/0002764211409384.

Marchezini M., 2015. The Biopolitics of Disaster: Power, Discourses, and Practices, Human Organization, $\mathrm{n}^{\circ}$ 4, vol. 74, p. 362-371. DOI: 10.17730/0018-7259-74.4.362. 
Mehnen N., Mose I., Strijker D., 2013. Governance and Sense of Place: Half a Century of a German Nature Park, Environmental Policy and Governance, $\mathrm{n}^{\circ}$ 1, vol. 23, p. 46-62. DOI: 10.1002/eet.1592.

Ministère de la Culture et des Communications (MCC), 2013. Gare ferroviaire de Lac-Mégantic. Consulté à l'adresse : [http://www.patrimoine-culturel.gouv.qc.ca/rpcq/ detail.do ? methode $=$ consulter\&id $=93405 \&$ type $=$ bien $]$.

November V., D’Alessandro-Scarpari C., Rémy É, 2004. Un lieu en controverse : une controverse qui fait lieu(x). Norois, nº 193, vol. 4, p. 91-102. DOI: 10.4000/norois.799.

Oliver-Smith A., Hoffman S.M., 1999. The Angry Earth. Disaster in Anthropological Perspectivxe, New York/London, Routledge, 334 p.

Raymond C. M., Brown G., Weber D., 2010. The Measurement of Place Attachement: Personal, Community and Environmental Connections, Journal of Environmental Psychology, $\mathrm{n}^{\circ}$ 4, vol. 30, p. 422-434. DOI: 10.1016/j. jenvp.2010.08.002.

Revet S., Langumier J. (dir.), 2013. Le gowvernement des catastrophes (coll. Recherches internationales), Paris, Karthala, coll. « Recherches internationales », 280 p.

Réserve internationale de ciel Étoilé du Mont-MéganTIC (RICEMM), s.d., Réserve internationale de ciel étoilé $d u$ Mont-Mégantic. Consulté à l'adresse : [http://ricemm.org/ reserve-de-ciel-etoile/].

Ritchie, L.A., Gill D.A., Picou J.S., 2012. The Exxon Valdez and BP oil spills, American Behavorial Scientist, $\mathrm{n}^{\circ} 1$, vol. 56, p. 3-23. DOI: 10.1177/0002764211408585.

Sмiтh S.G., 1994. The Essential Qualities of a Home, Journal of Environmental Psychology, $\mathrm{n}^{\circ}$ 1, vol. 14, p. 31-46. DOI: 10.1016/S0272-4944(05)80196-3.

Société des Établissements de Plein air du Québec (SÉPAQ), 2016, Parc national du Mont-Mégantic. Consulté à l'adresse : [http://www.sepaq.com/pq/mme/].
Trentelman C.S., 2009. Place Attachement and Community Attachement : a Primer Grounded in the Lived Experience of a Community Sociologist, Society and Natural Resources, ${ }^{\circ}$ 3, vol. 22, p. 191-210. DOI: 10.1080/08941920802191712.

Turner P., Turner S., 2006. Place, Sense of Place, and Presence, Presence: Teleoperators \& Virtual Environments, $\mathrm{n}^{\circ} 15$, vol. 2, p. 204-217. DOI: 10.1162/pres.2006.15.2.204.

Ullberg S., 2013. Ethnographier les mémoires des catastrophes. Terrain translocal à Santa Fe (Argentine), in Revet S., LANGUMIER J. (dir.), Le gowvernement des catastrophes, Paris, Karthala, coll. «Recherches internationales », p. 187-214.

Valiquette L'Heureux A., 2016. La tragédie de Lac-Mégantic et l'atrophie de la vigilance dans le secteur public. Thèse de doctorat en administration publique, École nationale d'administration publique, $333 \mathrm{p}$.

Ville de Lac-Mégantic, 2014. Réinventer la ville. Plan directeur de reconstruction du centre-ville de Lac-Mégantic, Rapport d'étape - octobre 2014. Consulté à l'adresse : [http://www.ville.lac-megantic.qc.ca/fileadmin/Documents/ ville-lac-megantic/Ville_de_Lac-Megantic/Actualites/ GTR_13305_rapportcameo-final_2014-10-28_LR.pdf].

Ville de Lac-Mégantic, 2013. Programme particulier d'urbanisme du centre-ville de Lac-Mégantic. Consulté à l'adresse : [http://fr.scribd.com/doc/228480758/PPU-Lac-Megantic].

Ville de Lac-Mégantic, 2012. Historique. Consulté à l'adresse : [http://www.ville.lac-megantic.qc.ca/la-ville/portrait-de-la-ville/historique/].

Williams D.R., 2002. Leisure identities, globalization, and the politics of place, Journal of Leisure Research. $\mathrm{n}^{\circ} 4$, vol. 34, p. 351-367. DOI: 10.1080/00222216.2002.11949976 